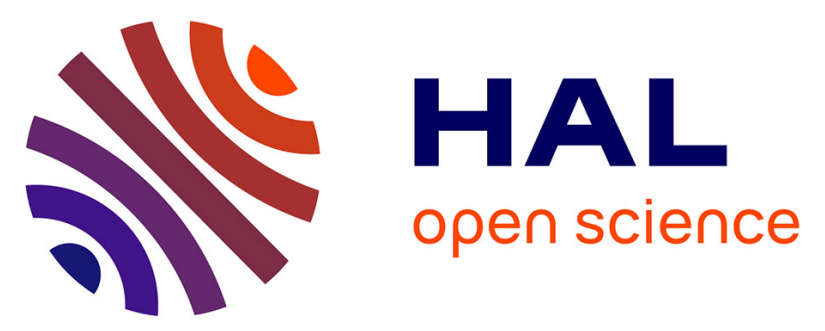

\title{
Beyond LoRa and NB-IoT: Proposals for Future LPWA Systems
}

Guillaume Vivier, Valérian Mannoni, Francois Dehmas, Vincent Berg, Lina

Mroueh, Yi Yu, Diane Duchemin, Jean-Marie Gorce, Julio Manco-Vasquez, Faouzi Bader

\section{To cite this version:}

Guillaume Vivier, Valérian Mannoni, Francois Dehmas, Vincent Berg, Lina Mroueh, et al.. Beyond LoRa and NB-IoT: Proposals for Future LPWA Systems. EuCNC 2019 - European Conference on Networks and Communications, Jun 2019, Valencia, Spain. pp.523-527, 10.1109/EuCNC.2019.8802007 . hal-02106227

\section{HAL Id: hal-02106227 \\ https://hal.science/hal-02106227}

Submitted on 22 Apr 2019

HAL is a multi-disciplinary open access archive for the deposit and dissemination of scientific research documents, whether they are published or not. The documents may come from teaching and research institutions in France or abroad, or from public or private research centers.
L'archive ouverte pluridisciplinaire HAL, est destinée au dépôt et à la diffusion de documents scientifiques de niveau recherche, publiés ou non, émanant des établissements d'enseignement et de recherche français ou étrangers, des laboratoires publics ou privés. 


\title{
Beyond LoRa and NB-IoT: Proposals for Future LPWA Systems
}

\author{
Guillaume Vivier ${ }^{(1)}$, Valérian Mannoni ${ }^{(2)}$, François Dehmas ${ }^{(2)}$, Vincent Berg ${ }^{(2)}$, Lina $\operatorname{Mroueh}^{(4)}$, \\ Yi Yu ${ }^{(4)}$, Diane Duchemin ${ }^{(3)}$, Jean-Marie Gorce ${ }^{(3)}$, Julio Manco-Vasquez ${ }^{(5)}$, and Faouzi Bader ${ }^{(5)}$ \\ (1) Sequans, Paris, France \\ (2) CEA Leti, Grenoble, France \\ (3) Univ Lyon, INSA Lyon, Inria, CITI, Villeurbanne, France \\ (4) ISEP, Paris, France \\ (5) CentraleSupélec, campus of Rennes, France
}

\begin{abstract}
This paper proposes areas of improvement for Low Power Wide Area (LPWA) solutions. After recalling the rationale of these systems, it summarizes the main challenges of existing solutions and proposes various improvements at PHY and MAC layer that were investigated in context of the EPHYL project. NB-IoT and LoRa were mostly considered in the analysis of existing techniques, while proposals for improvement address both waveform design and resource allocation strategy. We propose as well introduction of new paradigm such as network coding to benefit of the sparse characteristic of some LPWA use cases. Some of the proposals are validated experimentally in the FIT/CorteXlab environment, offering to the research community a framework to experiment and compare their improvements of LPWA systems.
\end{abstract}

Index Terms-LPWA, cellular IoT, waveform, radio access, NB-IoT, LoRa, FIT/CorteXlab

\section{INTRODUCTION}

Recent forecast [1] estimates that the number Internet of Things (IoT) connections will reach 22 billions worldwide, including cellular IoT as well as short range IoT. The latter one are nowadays most common and use local area network techniques such as Wifi, Zigbee or Bluetooth to connect to an access point (typically a smartphone or a box) to access the wider network. Over the past few years, new approaches often referred as Low Power Wide Area (LPWA) networking technologies have emerged from the shadow to provide direct connectivity between the objects and the Network. Proprietary solutions were defined such as Sigfox, LoRa or other [2], [3] while the cellular industry mandated the 3GPP to define optimized solutions for IoT. As a result, the LTE category 1 specifications were frozen offering a low-end LTE category for machine type communication (MTC), and two main lower categories were standardized in context of Release 13: LTE$\mathrm{M}$ and Narrow Band IoT (NB-IoT). These two categories were defined with aggressive objectives in terms of battery life, cost, extended coverage, while maintaining the benefits of cellular systems, such as security, coverage, quality of service or mobility. They are moreover evolving continuously to include further improvements and cellular technologies will connect more and more IoT, about 4 billions in 2027 according to $[1]$.
However, from theoretical standpoint, these LPWA systems apply very simple mechanisms to reliably transmit information. Physical layer and medium access techniques rely on well proven and somehow basic technologies. The spectral efficiency is relatively far from optimal and the energy per transmitted information bit is relatively high. Moreover, as the number of connected objects is expected to explode over the coming years, access to current generation of systems will be congested and new innovative technologies will be required, to improve the overall capacity of the cell or/and the data rate while providing similar level of power consumption at the IoT node.

Several technical and scientific barriers need to be addressed to allow a full global deployment of cellular network for efficient IoT access:

\section{- Barrier in the waveform design}

Current cellular technologies are based on a cellular concept: base stations broadcast pilots and other information to help the terminal for initial synchronization and to control the access to the radio resource. This approach, quite well adapted to mobile cellular terminals, may not be suited to low data rate IoT which may prefer to limit the overhead of synchronization for transmission, in order to minimize the overall power consumption. Research in wireless waveforms has been so far focused on getting closer to the Shannon limit for high data rate solutions (Broadcast, 4G). Current generation of LPWA systems heavily relies on repetition to improve sensitivity performance. Gain in spectral efficiency at low sensitivity is therefore desirable (see Fig. 3). Improvement should focus on the waveform design (OFDMA assumes strong synchronisation), the channel forward error correction (turbo codes are rather optimized for high data rates), or on the overall procedures (access, mobility support, retransmissions, etc.)

- Barrier in resource management in context of massive deployment of IoT

Cellular based LPWA solution relies on cellular concept: densification of deployment to provide capacity and centralized scheduling to optimize the resource al- 
location. They also operate in license spectrum bands. Sigfox or LoRa type of solution considers unlicensed spectrum and rather unsynchronized resource allocation: nodes transmits whenever they want and the network side should deal with possible collisions. For both approaches, whatever the optimization of the PHY layer, the general performance of an IoT network will rely on its capability to cope efficiently with interference and resource sharing. Clearly optimizing the PHY layer without considering its use at the radio resource management (RRM) side would not be efficient.

The need to introduce advanced MTC solutions to overcome the above listed barriers is not novel. Several papers were produced in the recent years, such as [4] which is providing a good overview of various possible improvements. One of the particularity of our work is that we prototype our proposed improvement on the open testbed FIT/CorteXlab [5] offering to the research community a framework to evaluate a cellular based IoT system.

The paper is structured as follows. Section II briefly analyses existing systems to highlight their limitations. Then, section III proposes possible enablers for future LPWA systems. Section IV introduces the prototyping environment and finally section $\mathrm{V}$ concludes and gives perspective.

\section{ANALYSis OF EXISTING LPWA SOLUTIONS}

\section{A. Analysis from PHY layer}

Some solutions have been proposed to meet the requirements on the Physical layer of the new LPWA networks i.e. long range (low sensitivity) and low power consumption (long battery life). NB-IoT, introduced in the latest LTE release [6], relies on this strategy. The uplink transmission of this solution combining the powerful Turbo Code (TC) [6] as Forward Error Correction (FEC) and a repetition factor guarantees QoS at low levels of SNR. DFT-spread OFDM (SC-FDMA) as well as single tone waveforms are part of the standard. This standard enables to reach the lowest sensitivities and offers a flexibility in data rate that makes it able to match numerous applications (Cf. Fig. 2). However, NB-IoT waveform has a significant Peak to Average Power Ratio (PAPR) which imposes high constraints on the Power Amplifier (PA) and increases energy consumption at the transmitter level (Tx). Another solution identified to address the LPWA requirements and to decrease the Tx power consumption is LoRa. This technology uses a proprietary and patented waveform based on chirps and called Chirp Spread Spectrum (CSS) [7]. CSS is an orthogonal modulation with a constant envelope and therefore has the good property of having a zero PAPR. It is then highly energy efficient at the transmitter side. This waveform is combined with a Hamming code. This kind of FEC being relatively poor, the resulting performance (as shown in Fig. 2) are then far from Shannon's theoretical limit. Thus, we proposed to use an alternative waveform: Turbo-Frequency Shift Keying (Turbo-FSK). It combines the advantages of the two waveforms described above [8]. Low levels of sensitivity are achieved thanks to the use of a combination of orthogonal modulation, a parallel concatenation of convolutional codes and an iterative receiver. The scheme also offers a constant envelope modulation which reduces the constraints imposed on the PA and the power consumption. While Turbo-FSK has solely been presented as an alternative to other standalone solutions for LPWA, the use of Frequency Shift Keying (FSK) modulation allows to integrate the technology in an OFDM framework.

\section{B. Analysis from MAC and RRM layer}

One of the main challenges of the LPWA networks planners is to appropriately estimate the bandwidth that meets the expected needs in an economical way. This radio dimensioning is highly related to the network parameters that include the devices traffic, the required QoS, the intensity of active devices and network coordinators, the wireless channel conditions, the equipments types, the antenna configurations and the network access protocol. The EPHYL project proposes in [9], [10] statistical tools derived from stochastic geometry to help the network planners to evaluate, adjust and adapt the allocated bandwidth according to the above network parameters. The average statistical behavior of the network is considered with active devices randomly distributed according to a Poisson Point Process marked by the wireless channel randomness. We have considered two types of networks: the centralized NBIoT like network and the non-coordinated network like LoRa network. In the NB-IoT, the access to the shared medium is managed by a central coordinator. In LoRa network, the access to the shared medium is completely random and managed by an Aloha-based MAC protocol. The proposed analytic model highlights and quantifies the radio resources gain obtained when deploying multiple receiver at the collector side. Figure 1 illustrates the required number of radio resources (RR) to allocate with different collectors intensities in a NB-IoT context. As it can be seen, the statistical model quantifies the gain in terms of RR when exploiting receiver diversity.

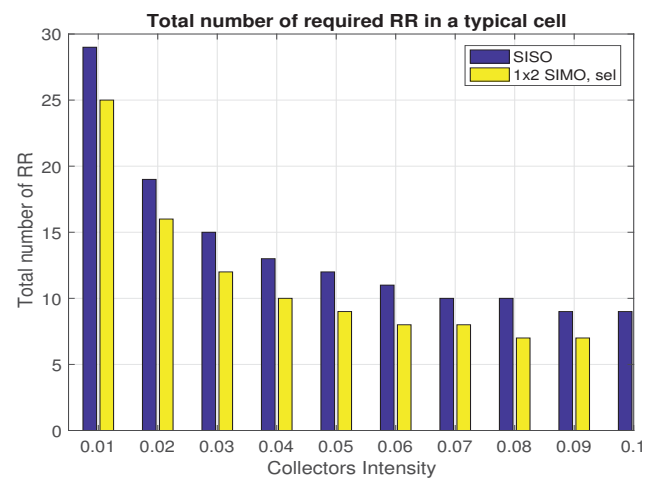

Fig. 1. Required Total Number of Radio Resource in a Typical Cell

\section{Main limitations from existing systems}

NB-IoT relies on cellular approaches, thus inherits from the benefit and the drawback of cellular systems. With respect 
to LPWA service, a possible drawback is the need to be synchronized with the network prior to anything (transmit or receive). Although the 3 GPP has defined an "early data transmission" in Release 15 [11], by reducing the handshake mechanism from 5 to 3 steps thus allowing to transmit data without being in the connected mode, the device still has to spend some time and energy to retrieve the network synchronization prior to send a message. Another possible limitation of NB-IoT comes from the use of repetition for very challenging channel conditions (MCL up to $160 \mathrm{dBm}$ ). More efficient modulation and coding techniques may be considered for such hard conditions. So, NB-IoT can be improved from the waveform perspective, while in the following we raise some concerns about the scalability of LoRA system.

Although the LoRa networks can have a coverage range of $22 \mathrm{~km} \mathrm{[2],} \mathrm{the} \mathrm{distribution} \mathrm{of} \mathrm{the} \mathrm{nodes} \mathrm{reaching} \mathrm{the} \mathrm{cell}$ capacity is highly in-homogeneous under optimal Aloha access assumptions, taking into account the base station sensitivity and suburban channel attenuation [12]. This leads to a high concentration of devices at the center of the cell, especially those with high traffic needs (with an actual rate restricted to $2 \mathrm{kbit} / \mathrm{s}$ due to the obligatory ACK reception before the next message). The authors also show that less than $10 \%$ of the devices (at the cell capacity) can be located farther than $5 \mathrm{~km}$ away from the gateway, which limits the scalability of LoRa networks. They thus infer that LoRa is operable for moderately dense networks, with very low traffic without high requirement on the latency nor on the reliability. Furthermore, the scalability is also limited by the co-spreading sequence interference. As mentioned in [2], [13], the rejection factor of two signals with different spreading factors is large enough to consider they are orthogonal. However this is not the case for the same spreading factor and [13] show that the coverage probability decays exponentially with the user density of a cell. E.g. for the simulation parameters, in a $12 \mathrm{~km}$ radius cell, from approximately 350 users, the co-spreading sequence interference is the main cause of outage. Moreover, the gateways can only process up to 9 simultaneous signals in parallel (where a signal is characterised by its spreading factor and the channel picked out of the 3 to 9 deployed channels). It thus makes LoRa to seem not really well suited for high access demand as required for massive MTC, and implies low performance in terms of capacity and reliability but also latency and energy efficiency since devices have to retransmit their messages.

The next section provides an overview of improvements proposals for future LPWA systems, in order to mitigate the limitations briefly identified.

\section{ENABLERS FOR FUTURE LPWA SYSTEMS}

\section{A. 3GPP proposals}

The 3GPP standardisation continuously improves the cellular technologies. As such, Cat-M and NB-IoT are improving with the releases, since their introduction in Rel. 13. Hence, Rel. 14, and Rel. 15 included their bunch of enhancement, mostly focusing on better system spectral efficiency and lower power consumption for the device. In 2019, standardisation

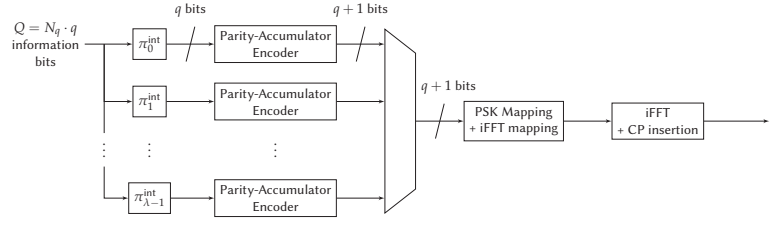

Fig. 2. Transmitter architecture of Turbo-FSK

focuses on completing release 16 and starting Rel. 17. While 3GPP introduced a new waveform in Rel. 15 for $5 \mathrm{G}$ (so called "New Radio"), it has been decided that LPWA use cases will be served by Cat-M and NB-IoT and their evolutions. As a result, there is no plan in 3GPP to define a new LPWA waveform in context of $5 \mathrm{G}$, but rather to ensure good coexistence or complementarity of cat-M, NB-IoT within 5G deployments.

\section{B. Turbo-FSK}

Turbo-FSK waveform introduced by Roth et al. and described in [8] combines an orthogonal modulation FSK with a powerful channel coding based on the Turbo principle. The main motivation of this scheme is to operate close to the Shannon capacity while using a constant envelop modulation (or a PAPR close to OdB). The structure of the Turbo-FSK transmitter, illustrated in Fig. 2, is composed of $\lambda$ repetition stages. Each stage is then a differently interleaved version of the same input information bit sequence and fed to a simple parity accumulator. The resulting coding bits are then mapped onto a Turbo-FSK codeword: a combination of a linear $N_{L^{-}}$ point phase shift keying (PSK) modulation and a non-linear $N_{\perp}$-carrier FSK modulation [8].

The Turbo-FSK receiver relies on the fundamental principle of a turbo decoder. For each stage, the receiver estimates the information bits given both the observation of the channel for this version and the a priori information coming from the decoding of the other versions. To do so, a modified version of the usual BCJR (Bahl, Cocke, Jelinek and Raviv) algorithm [14] is used on the trellis describing the code. The algorithm computes the a posteriori (APP) probability of the codewords given the observation and deduces the APP of information bits. One iteration consists then in doing this process for each stage and multiple iterations are required.

Fig. 3 shows the performance of the Turbo-FSK waveform with the evolution of the spectral efficiency for different configurations $\left(\lambda, N_{L}, N_{\perp}\right)$ as the function of the required $E_{b} / N_{0}$ to reach a $\mathrm{BER}=10^{-4}$ [8]. These results clearly demonstrate that the Turbo-FSK fulfills its original purpose and exhibits promising performance for the low spectral efficiencies compared to NB-IoT or LoRa.

\section{Indexed modulation}

Index modulation (IM) has been proposed for $5 \mathrm{G}$ wireless networks for the design of downlink transmission protocols [15]. However, we believe that applying IM to the uplink cellular IoT standards is more relevant, since the complexity is offloaded from end devices and kept at the receiver. This 


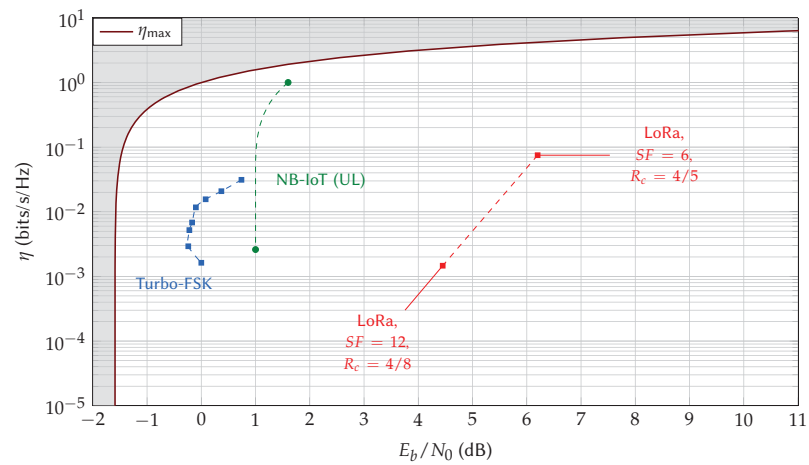

Fig. 3. Performance of the Turbo-FSK waveform versus the maximum achievable spectral efficiency and comparison with NB-IoT and LoRa

application is also motivated by the consideration that IM is more efficient in low modulation orders and for low number of carriers. We have introduced the principle of single carrier Frequency Division Multiple Access scheme using indexed modulation (SC-FDMA-IM) for transceiver for uplink NBIoT transmissions as detailed in [16] (see presented scheme in Fig 4), where a user $u$ (from a total of $U$ active users) has $m_{u}$ bits to transmit introduced to the index modulation block. IM processing for a user $u$ is based on the following parameters: $G_{u}$ is the number of groups within an SC-FDMAIM block symbol of duration $T, n_{u}$ is the number of subcarriers in each group, and $k_{u}$ is the number of active subcarriers in each group. In our case, $k_{u}$ is supposed to be fixed for every group and during the transmission in order to reduce the complexity. Provided performances relate to IM schemes

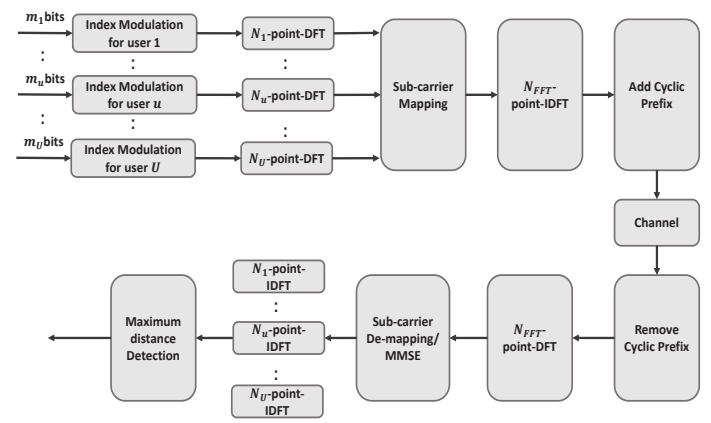

Fig. 4. SC-FDMA-IM transceiver for NB-IoT transmissions

using SC-FDMA for uplink NB-IoT transmissions include both localized and interleaved multiple users access modes. In [16], for the same spectral efficiency as classical SC-FDMAIM, we have showed that index modulation can lead to a gain of $50 \%$ in terms of energy efficiency while maintaining an affordable transceiver complexity. Due to favourable spectrum spacing resulting from IM, the BER in Fig 5 achieves a gain of $3.5 \mathrm{~dB}$ in terms of $\mathrm{Eb} / \mathrm{N} 0$ for $\mathrm{BER}=10^{-4}$ for $\mathrm{NB}-\mathrm{IoT}$ interleaved mode and $3 \mathrm{~dB}$ in the localized mode at the cost of a PAPR increase. Thus PAPR reduction techniques need

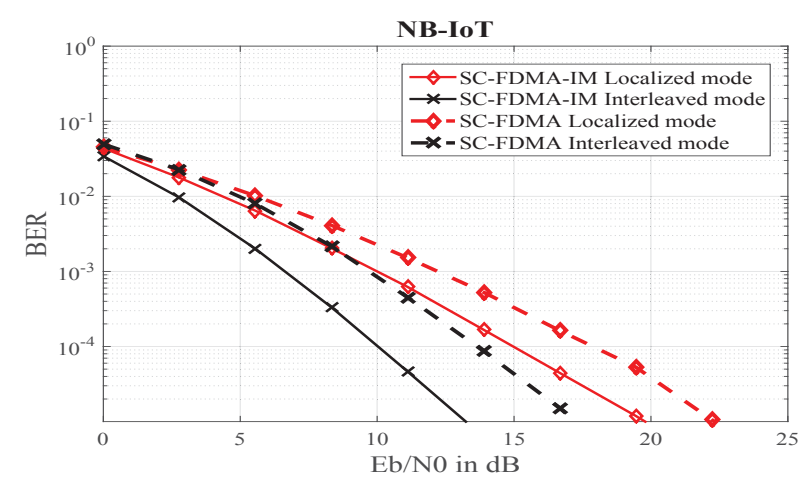

Fig. 5. BER performance for SC-FDMA-IM in NB-IoT scenario

to be used. These significant results encourage more studies about SC-FDMA-IM, especially for IoT applications.

\section{Efficient repetition based on Polar codes}

In a LoRa-Like network, the access to shared medium is managed by an ALOHA multiple access protocol. The long communication ranges as well as the high level of interference induce significant packet loss and affect highly the network reliability. Unlike cellular networks, the LPWAN communications are mostly uplink and the use of Automatic Repeat Request (ARQ) protocol is not efficient. To address this challenge, low complexity repetition schemes that benefit from the time-varying channel condition are widely used. In this case, successful data recovery requires a significant channel variation from a bad to a good state depending on the data rate. This induces a relatively high decoding latency especially for slow time-varying channel. The EPHYL project proposes to reduce the packet recovery latency by exploiting multiple channel states defined with respect to various transmission data rates, that are enabled by a rate-less polar code. We characterize the good channel sequence state of this code, and evaluate its latency performance in a slow and fast-time varying context. The numerical results show the efficiency of the rate-less polar code scheme in a slow time varying channel compared to the repetition code.

\section{E. NOMA based direct transmission}

In order to reduce the energy spent for resource allocation prior to data transmission, we aim at proposing a solution for direct data transmission, in unconnected mode like Aloha, based on a single random access while allowing resource sharing for many users, so that the collisions and bottleneck effect of massive random access are highly reduced. In [10] we have introduced a random coded non orthogonal multiple access (NOMA) scheme based on Gaussian coding, whose each code is uniquely associated to a user and allows to perform the resource sharing while restricting the active users interference and acting as identifiers for the user detection. This solution is inspired by the Compressed Sensing frameworks [17]. On the receiver side, dedicated algorithms are needed to take advantage of the assumed sparse user activity. However, to be 
able to efficiently retrieve the user activity and their messages, existing receiver algorithms such as NBOMP [18] require a high level of prior knowledge, which is incompatible with our goal of direct transmission with low coordination overhead. We therefore have defined optimal and optimal-based detectors (e.g. It-MAP in Fig. 6) to cope with the unknown channel impulse response of the users or the unknown active user subset size, so that it avoids to transmit coordination messages before the user can send its data.

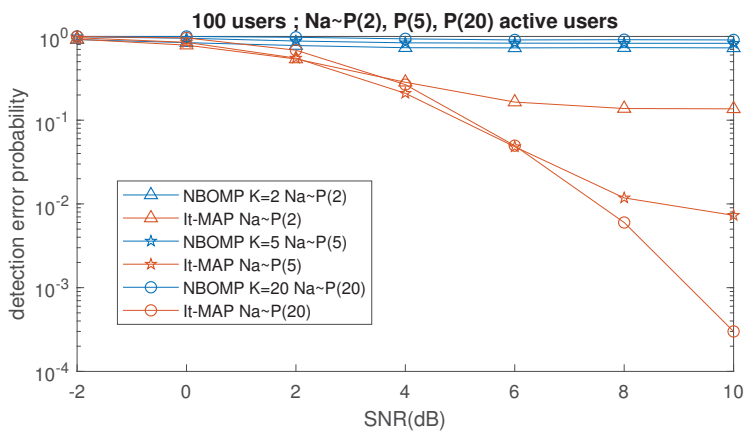

Fig. 6. Detection Error Probability of It-MAP and NBOMP for unknown active subset sizes $N a(N a \sim \mathcal{P}(\Lambda)$ with $\Lambda=2,5,20)$

\section{PRototyping ENVIRONMENT}

FIT/CorteXlab [5] is a large scale radio testbed composed of an extensive set of SDR nodes. These latter provide substantial RF flexibility, enabling to tune the operating frequency, the channel bandwidth, the emitted power and the waveform used to communicate. The testbed is located in a large shielded room which is partially covered with electromagnetic absorbing foams. Conclusively, the design of the room enables to ensure a high level of reproducibility of experiments. Last but not least, all SDR nodes are remotely accessible through a service interface allowing to deploy proprietary solutions. FIT/CorteXlab radio testbed thus has the key properties to develop an IoT network framework, remotely and freely available for the research community. It allows to fasten the execution of large scale emulated radio experiments, such as the one developed for the prototyping of the above mentioned proposals.

More specifically, we provide a customizable and open source design for IoT networks prototyping in a massive multiuser, synchronized and reproducible environment. The massive access feature is managed by emulating several sensors per radio nodes. Two categories of network components are used in our design: a base station unit and a multi-sensor emulator unit. The components are separately hosted in dedicated and remotely accessible radio nodes. The base station and emulated sensor nodes are designed such that it is possible to add/parameterise the functional blocks embedding the investigated PHY and MAC proposals, while still working on the same time-base, thanks to the connection of the SDR nodes to 8-Channel Clock Distribution modules, and synchronizing signals broadcasted by the base station. The framework allows for extensive testing with various communication use-cases and with the possibility to adapt the aggregated functional blocks depending on the desired performance analysis.

\section{Conclusion AND PERspective}

In this paper, we discussed the main limitations of existing LPWA systems and proposed some targeted improvements at MAC and PHY layer. A key aspect of our work in the EPHYL project is that most of the innovative proposals are implemented in context of FIT/CorteXlab and will be made openly available to the research community.

\section{ACKNOWLEDGMENT}

This work has been supported by the French Agence Nationale de la Recherche (ANR), under grant agreement ANR-16-CE25-0002 (project EPHYL).

\section{REFERENCES}

[1] Ericsson, "Ericsson mobility report," November 2018. retrieved Feb. 2019 from https : //www.ericsson.com/en/mobility - report.

[2] C. Goursaud and J. M. Gorce, "Dedicated networks for IoT: PHY / MAC state of the art and challenges," EAI Endorsed Transactions on Internet of Things, vol. 1, p. 150597, oct 2015.

[3] U. Raza, P. Kulkarni, and M. Sooriyabandara, "Low power wide area networks: An overview," IEEE Communications Surveys Tutorials, vol. 19, no. 2, pp. 855-873, 2017.

[4] C. Bockelmann, N. K. Pratas, G. Wunder, S. Saur, M. Navarro, D. Gregoratti, G. Vivier, E. De Carvalho, Y. Ji, C. Stefanović, et al., "Towards massive connectivity support for scalable mmtc communications in $5 \mathrm{~g}$ networks," IEEE Access, vol. 6, pp. 28969-28992, 2018.

[5] L. S. Cardoso, O. Oubejja, G. Villemaud, T. Risset, and J. M. Gorce, "Reliable and Reproducible Radio Experiments in FIT/CorteXlab SDR Testbed: Initial Findings," in Crowncom, (Lisbon, Portugal), 2017.

[6] 3GPP, "3rd Generation Partnership Project; Technical Specification Group Radio Access Network; Evolved Universal Terrestrial Radio Access (E-UTRA); Physical layer procedures, ," tech. rep. Release 15, 3GPP TS 36.213, v 15.2.0. June 2018.

[7] Semtech, "Lora modulation basics," tech. rep. v 2, May 2015. https : //www.semtech.com/uploads/documents/an1200.22.pdf.

[8] Y. Roth, The physical layer for low power wide area networks : a study of combined modulation and coding associated with an iterative receiver. Theses, Université Grenoble Alpes, July 2017.

[9] M. T. L. Mroueh, Y. Yu and P. Martins, "Statistical uplink dimensioning in licensed cellular low power iot network," in IEEE ICT, IEEE, 2018.

[10] M. Chafii, C. F. Bader, F. Dehmas, D. Duchemin, L. Mroueh, and G. Vivier, "Intermediate results on RRM enablers for massive and dense IoT networks,", tech. rep. Oct. 2018.

[11] 3GPP, "Further NB-IoT enhancements (RP-171428)," 2017.

[12] K. Mikhaylov, J. Petäjäjärvi, and T. Hänninen, "Analysis of Capacity and Scalability of the LoRa Low Power Wide Area Network Technology," 22th European Wireless Conference, pp. 119-124, 2016.

[13] O. Georgiou and U. Raza, "Low Power Wide Area Network Analysis: Can LoRa Scale?," IEEE Wireless Communications Letters, vol. 6, no. 2, pp. 162-165, 2017

[14] L. Bahl, J. Cocke, F. Jelinek, and J. Raviv, "Optimal decoding of linear codes for minimizing symbol error rate (corresp.)," IEEE Transactions on Information Theory, vol. 20, pp. 284-287, March 1974.

[15] E. Basar, "Index modulation techniques for $5 \mathrm{~g}$ wireless networks," IEEE Communications Magazine, vol. 54, no. 7, pp. 168-175, 2016.

[16] M. Chafii, F. Bader, and J. Palicot, "SC-FDMA with Index Modulation for M2M and IoT Uplink Applications," in IEEE Wireless Communications and Networking Conference (IEEE WCNC'2018), (Barcelona, Spain), Apr. 2018.

[17] A. K. Fletcher, S. Rangan, and V. K. Goyal, "OnOff Random Access Channels: A Compressed Sensing Framework." https://arxiv.org/pdf/0903.1022v2.pdf, 2009.

[18] R. Xie, H. Yin, X. Chen, and Z. Wang, "Many Access for Small Packets Based on Precoding and Sparsity-Aware Recovery," IEEE Transactions on Communications, vol. 64, pp. 4680-4694, nov 2016. 